\title{
A New Paradigm to Study the Influence of Attentional Load on Cortical Activity for Motor Preparation of Step Initiation
}

Aurore Braquet ${ }^{1 *}$, Madli Bayot ${ }^{*}$, Céline Tard $^{2}$, Luc Defebvre $^{2}$, Philippe Derambure ${ }^{1}$, Kathy Dujardin ${ }^{2}$, Arnaud Delval ${ }^{1}$

1 Univ. Lille, Inserm U1171-Degenerative and Vascular Cognitive Disorders, 59000 Lille, France; Department of Clinical Neurophysiology, Lille University Medical Center, 59000 Lille, France.

2 Univ. Lille, Inserm U1171-Degenerative and Vascular Cognitive Disorders, 59000 Lille, France; Department of Neurology and Movement Disorders, Lille University Medical Center, 59000 Lille, France.

Corresponding author:

Arnaud Delval, Neurophysiologie Clinique, Hôpital Salengro, Centre Hospitalier Universitaire, F-59037 Lille cedex, France

Tel.: + 33-320-446-462

Fax: + 33-320-446-355

E-mail: arnaud.delval@ chru-lille.fr

http://orcid.org/0000-0003-0642-3541

* First co-authors 


\begin{abstract}
Objective: Motor programme for gait initiation can vary as a function of attentional resources. The objective of the present study was to determine whether alertness, orientation and executive control can modulate cortical activation during step initiation.
\end{abstract}

Methods: The attention network test (ANT) was used to control the influence of different attentional components on kinetic characteristics of step initiation and the associated cortical activity. Thirty healthy adults performed ANT combined with step initiation. The step execution time (SET) and anticipatory postural adjustments (APAs) were recorded. Movement-related cortical potentials (MRCPs) and eventrelated spectral perturbations (ERSPs) after response emission were analysed according to the presence or absence of cueing or conflict resolution.

Results: Step reaction time and thus SET were significantly shorter with cueing, whereas APA duration and SET were longer during conflict resolution. Moreover, alertness was related to a higher rate of anticipated responses, and conflicting situations were associated with a greater amount of multiple APAs. Attentional load did not affect MRCPs but ERSPs: trials with a cue showed earlier posterior alpha and beta desynchronisations before APA onset. Furthermore, we found earlier, more pronounced and longer alpha- and beta-band desynchronisations over the sensorimotor cortex for trials with incongruent flankers.

Conclusion: Our results showed that attention has an impact on step initiation. A specific pattern of response-locked ERSPs seems to mirror behavioural effects of attentional load on step initiation. This new paradigm combining ANT and step initiation is therefore promising to investigate the interaction between attention and gait initiation in pathological populations.

Keywords: Attention Network Test, Step initiation, Electroencephalography, Anticipatory postural adjustments 


\section{Introduction}

Gait initiation is a motor programme whose characteristics can vary as a function of the attentional resources (Uemura et al. 2013a; Tard et al. 2013, 2016). They are known to modulate anticipatory postural adjustments (APAs) before movement execution (Lajoie et al. 1996; Delval et al. 2012; de Souza Fortaleza et al. 2017). Indeed, gait initiation requires more attentional resources than gait (Suzuki et al. 2004, 2008) and may cause more dual-task interference with an attentional task than steady-state walking does (Uemura et al. 2012). For instance, errors in motor programming have been exhibited in tasks requiring executive control (Uemura et al. 2013a; Delval et al. 2018), and particularly in elderly subjects (Cohen et al. 2011; Sparto et al. 2014). In fact, gait initiation is executed in two phases: the preparation phase (corresponding to APAs) and then the execution phase (corresponding to the time interval between "toe-off" and "heel strike" for the swing leg). During APAs, the centre of pressure $(\mathrm{CoP})$ is displaced according to a stereotypical pattern: first backwards and towards the swing leg, and then towards the stance leg (Brenière and Do 1991; Jian et al. 1993).

APAs can be modulated by attentional alerting (Tard et al. 2013) and orienting (Tard et al. 2016) processes. Indeed, when a cue preceded the gait initiation signal, we observed that early APAs occurred more frequently (Tard et al. 2013). Moreover, indicating the direction of the subsequent target which will state the required foot to initiate gait had a beneficial effect on step execution time (that includes earlier APAs as well as the subsequent step) (Tard et al. 2016). Cohen et al. (2011) and Uemura et al. (2013a) have also shown that when subjects are in a conflict situation, APAs can be first executed on the wrong side and then lengthen the step latency. This constitutes "APA errors". Attention can modulate these APA errors for orientation mechanisms (Tard et al. 2016). Healthy subjects can also exhibit steps with several APAs before being able to perform the step execution phase. This kind of abnormal APAs together with APA errors will be named "multiple APAs" in the present paper (as in (Cohen et al. 2017)).

Posner and Petersen (1990; 2012) proposed a three-network model of visuospatial attention: alerting, orientation and executive control. These three networks, although anatomically separated and with uncorrelated efficiencies (Fan et al. 2002), may interact amongst themselves (Fan et al. 2009). Achieving and maintaining an alert state is the usual definition of alertness, orientation consists in the selection of relevant information from sensory inputs, whereas executive control is involved in monitoring and solving potential conflicts between contradictory responses. The latter network can be evaluated by a flanker task for example (Cohen et al. 2011). All different aspects of visuospatial attention can be investigated by the now widely used Attention Network Test (ANT), which allows independent assessment of alertness, orientation and executive control, as well as their interaction (Fan et al. 2002). Usually, ANT is administered with a response by manual pressing on a computer keyboard. 
Since Posner and Petersen model's description, several studies have evaluated the cortical and behavioural characteristics of each attentional network (Fan et al. 2002; Gamboz et al. 2010): in these behavioural studies, reaction time (RT) was shorter when a neutral cue was presented before the target compared to the situation without cueing ("alerting effect"). It was also shorter in presence of a spatial cue than in presence of a neutral cue ("orienting effect"). Likewise, RT was longer with incongruent than with congruent flankers ("conflict/executive control effect"). Few studies have evaluated the influence of attentional capacities on step initiation (Cohen et al. 2011; Uemura et al. 2013a, b; Tard et al. 2013, 2016). The step initiation task is of particular interest because it is known to reflect early mechanisms of motor preparation that are not shown by analysing simple reaction times (Tard et al. 2016). The choice of investigating gait initiation can also be easily understood when we know the ability to take a quick step represents an important motor skill that can prevent a fall from occurring, regardless of the nature of the fall, in older adults as well as in patients with Parkinson's disease and freezing of gait (Lord and Fitzpatrick 2001; van den Bogert et al. 2002).

The cerebral functional substrates of standard ANT have been namely explored with event-related potentials (ERPs) based on electroencephalographic (EEG) recordings (Fan et al. 2007; Neuhaus et al. 2007, 2010; Deiber et al. 2013; Galvao-Carmona et al. 2014; Williams et al. 2016). In young adults, Williams et al. found a larger fronto-central target N200 for incongruent targets compared to congruent targets (Williams et al. 2016), whereas Neuhaus et al. (2007) did not emphasise any relationship between the amplitude of N200 and target congruency. As a reminder, N200 component was proposed either to mirror the recruitment of top-down resources to improve stimulus evaluation when conflict is detected or to reflect an evaluation of the possible outcomes rather than conflict detection (Williams et al. 2016). Fronto-parietal P300 modulations during executive control have also been reported (except in (Fan et al. 2007; Williams et al. 2016)), with frontal P300 increment and parietal P300 decrement that may mirror respectively, on the one hand, response withholding, prepotent response inhibition and correct response execution related to incongruent flankers (via frontal "nogo P300") (Neuhaus et al. 2010) and, on the other hand, increased difficulty of visual target detection as well as inhibition in the condition with an incongruent target (via parietal "P300b") (Neuhaus et al. 2007, 2010; Deiber et al. 2013; GalvaoCarmona et al. 2014). Additionally, peak latency of target $P 300$ was longer for incongruent targets than for congruent ones at centro-parietal and fronto-central sites, reflecting the use of more time to evaluate the incongruent target (Neuhaus et al. 2007; Williams et al. 2016). Some studies have also measured the event-related spectral perturbations (ERSPs) during standard seated ANT (Fan et al. 2007; Deiber et al. 2013): in particular, the response-locked spectral analysis of conflict effect (i.e. the time-frequency pattern related to incongruent minus congruent target conditions) involved event-related desynchronisation (ERD) in theta and alpha bands before response onset and theta- and beta-bursts near or after response (Fan et al. 2007). Similarly, in the context of continuous mobilisation of the lower limbs, attention was related to lower power in theta, alpha and beta bands over the motor and 
somatosensory cortex and other more widely distributed cortical areas compared to mind wandering (Melinščak et al. 2014; Melinscak et al. 2016).

So far, no study has assessed ERPs nor ERSPs associated with an attentional paradigm like the ANT in a step-initiation task. Characteristics of cortical activation in a conflict or non-conflict situation task with or without cueing during step initiation remains to be better characterised. Additional knowledge at the cortical level regarding the interaction between attention and step initiation would therefore allow for a better pathophysiological understanding of public health issues associated with an impaired initiation of gait (e.g. falls and Parkinson's disease with freezing of gait). The new paradigm used in the current study combines ANT and step initiation and, consequently, only differs from the standard ANT by the motor task. That is the reason why, next to the stimulus-locked ERP and ERSP analyses that allow to track the sensory and cognitive stages of information in time, it is mainly interesting to lock EEG epochs to the motor response in order to study the activity of motor-related regions in the stages preceding and following action. Such ERPs are called movement-related cortical potentials or MRCPs and are comprised of four components: early Bereitschaftspotential/readiness potential (BP), late BP, negative motor potential (MP) and re-afferent potential/positive post-movement potential (RAP) (Shibasaki 2012; Berchicci et al. 2016). Motor-related ERSPs are alpha and beta ERD/S that represent respectively increased/decreased activation of cortical areas involved/not involved in the task (Pfurtscheller 1999). In the preparation phase toward the movement and during the movement itself, alpha and beta ERDs occur predominantly over the somatosensory cortex and the motor cortex, respectively. Then, after the termination of movement, ERSs are observable in the beta band (Pfurtscheller 1999; Shibasaki and Hallett 2006). The relevance of analysing response-locked EEG correlates with our paradigm relies on the fact that ERD/S and MRCP differ according to the type of movement (Vidailhet et al. 1993; Pfurtscheller et al. 1997, 1998; Jankelowitz and Colebatch 2002; Shibasaki and Hallett 2006). The ones related to the ANT carried out while standing will therefore specifically characterise step initiation in comparison with the standard ANT and its finger pushing on a computer keyboard.

The primary objective of the present study was to establish whether or not attentional load modification (using the ANT (Fan et al. 2002)) can modulate cortical activation during gait initiation through the analysis of response-locked ERPs and ERSPs. The study's secondary objective was to validate the current paradigm combining ANT and step initiation by obtaining outputs similar to those of the standard button-pushing-based ANT, i.e. behavioural results and cortical activation related to the influence of cueing on responses, target perceptual discrimination, information processing, inhibition of a prepotent response and execution of the correct response. This secondary aim is addressed in Online Resource 1.

Our hypotheses regarding ERPs and ERSPs were that activation of attentional networks (mainly frontoparietal areas for the conflict resolution network, frontal and parietal regions, particularly of the left 
hemisphere, for the alerting network, and parietal sites and frontal eye fields for the orienting network (Fan et al. 2005; Raz and Buhle 2006; Cohen and Ridderinkhof 2013)) would modify cortical preparation of movement. Indeed, a conflict situation could affect either the Bereitschaftspotential or alpha/beta ERD, with a smaller BP and a more pronounced and longer ERD for the condition with incongruent flankers. We expected differences in ERSPs in these EEG bands that are either sensitive to motor preparation (Pfurtscheller 1999) or attentional tasks (Fan et al. 2007). Similarly, cueing before target presentation could lead to a decreased latency of BP as well as an earlier ERD. As an additional assumption, in the ANT's conflict resolution condition, we would observe a higher proportion of multiple APAs - reflecting the early control of movement preparation, whereas the presence of a cue would induce an earlier step reaction time.

\section{Material and methods}

\subsection{Participants}

Thirty healthy adult volunteers (16 females, 14 males; 29 right-handed; mean (standard deviation) age: 39.4 (14.2)) participated in the study after providing their informed written consent. According to selfreport data, none of the participants had a history of medication use or disease that could interfere with gait. The study was approved by the local independent ethics committee (CPP Nord-Ouest, Lille, France; reference: 2015-A00013-46).

\subsection{Experimental setting}

\subsubsection{The attentional task}

The attentional task (Fig. 1) was an adaptation of the ANT (Fan et al. 2002). In our modified version combining an attentional load with the initiation of a step, the participant was instructed to stand centrally on a force platform, in a comfortable and stable posture, with his/her feet parallel and apart from few centimetres. A computer display screen was placed 1 meter in front of the subject. By analogy with the seated ANT paradigm, the participant was instructed to initiate a forward step as fast and as accurately as possible after presentation of a visual target in order not to prioritise an improvement of either reaction time or proportion of multiple APAs (Uemura et al. 2013b). Besides, he/she was told not to initiate gait during the presentation of a cue.

More particularly, the participant was instructed to stare at a cross located at the centre of the screen. A cue could then appear for $100 \mathrm{~ms}$ : either a central cue (central star), or a spatial cue (a star located at the right or left of the fixation cross). In the "valid cue" condition, the spatial cue was located where the target (a central arrow pointing to the right or left side) was going to point to, whereas in the "invalid 
cue" condition, the spatial cue was positioned on the opposite side. In more detail, the spatial cue was presented in the form of an asterisk positioned $7 \mathrm{~cm}$ to the right or left of the central fixation cross (corresponding to a visual angle of between $12^{\circ}$ and $15^{\circ}$ of eccentricity for central vision). The lack of a cue constituted the "no cue" condition. After a variable duration (from 100 to $700 \mathrm{~ms}$ ), the target appeared for $1500 \mathrm{~ms}$. The latter was presented centrally, at the level of the fixation cross, and was surrounded by flankers, either congruent or incongruent (Fig. 1). If the target appeared to point to the left, the participant had to initiate a step with his/her left foot; conversely, if the target was directed to the right, the participant had to start walking with his/her right foot. Subsequently, the fixation cross reappeared for a random period ranging between 1500 and $2500 \mathrm{~ms}$ after the target presentation.

The participant was instructed to step. Indeed, the assessment of step initiation and not gait initiation represents a limit of the current study. However, participants often performed a second step and, in any case, APAs during step initiation are similar to the ones carried out during gait initiation, except for the COP backward shift which is more ample during gait initiation. Finally, a good reason to having chosen to study step initiation instead of gait initiation is the nature of the ANT as a paradigm with time pressure. The combination of ANT with step initiation therefore allows to keep this time pressure that is essential for the comparison with the standard ANT.

Overall, four blocks of 75 trials each - separated by short breaks - were performed. Through those experimental blocks, each cue and target condition was presented in a pseudo-randomised order: 156 congruent trials, 144 incongruent trials, and 49, 61, 142 and 48 trials corresponding respectively to no cue condition, central cue condition, and both valid and invalid spatial cue conditions.

While previous studies using standard seated ANT assessed the reaction time for key press, the behavioural reaction time in the context of our paradigm combining ANT and step initiation is the step execution time ( $S E T$, i.e. the time interval between target presentation and heel contact of the swing foot (Uemura et al. 2013a)). The median SET was therefore calculated for each cue and target condition for each subject. Trials without any step, with an incorrect response (i.e. step initiation with the wrong foot) or an anticipated response (i.e. with a reaction time or the time interval between the appearance of the target and APA onset, $R T<100 \mathrm{~ms}$ ) were excluded from the analyses. It is noteworthy that, after exclusion of trials without any step, with an incorrect response or with an anticipated response, subjects presented trials that were distributed as follows (median (first quartile - third quartile)): 113 (104.25 126.25) congruent trials, 108.5 (89 - 116.5) incongruent trials, and 40 (32.25 - 42), 42 (36.25 - 47), 106 (93.5 - 114.75), 35 (31 - 38.75) corresponding respectively to no cue condition, central cue condition, and both valid and invalid spatial cue conditions. Subsequently, the different attentional networks defined by Posner and Peterson were evaluated in this way:

Alerting effect $=$ SET without cue - SET with central cue 


$$
\begin{aligned}
& \text { Orienting effect }=S E T \text { with central cue }- \text { SET with valid spatial cue } \\
& \text { Executive control effect }=\text { SET with congruent flankers }- \text { SET with incongruent flankers } \\
& \text { Validity effect }=\text { SET with invalid spatial cue }- \text { SET with valid spatial cue }
\end{aligned}
$$

It has to be noted that both incorrect and anticipated steps were quantified for each cue and target condition and each subject.

\subsubsection{Motion analysis}

Data were collected with a three-dimensional motion analysis system (VICON 370 ${ }^{\circledR}$, Oxford Biometrics, Oxford, UK), using eight infrared cameras sampled at $100 \mathrm{~Hz}$. Centre of pressure was measured with two force platforms (the ORG-5 model from AMTI ${ }^{\circledR}$, Watertown, MA, USA) sampled at $1000 \mathrm{~Hz}$. Reflective markers were placed on precise, reproducible anatomical landmarks on each foot: the toe (the head of the second metatarsal), the lateral malleolus and the heel. Data were then computed by the same operator using an in-house MATLAB ${ }^{\circledR}$ script (The MathWorks, Natick, MA, USA). The SET and its components were assessed by controlling, from the appearance of the target to heel contact, the vertical forces exerted on the force platform as well as kinematic features obtained with the 3D motion analysis system (Fig. 2). The SET includes:

- the reaction time $R T$, i.e. the time interval between the appearance of target $S 2$ and APA onset $T_{0}$.

- the anticipatory postural adjustments APAs. The APA duration was assessed by subtracting $\mathrm{T}_{0}$ from the toe-off time. The direction of the APA was considered to be normal if the CoP moved backwards and laterally towards the swing foot. Multiple APAs were defined as either an incorrect CoP displacement or unstoppable CoP displacement leading to several APAs. In case of multiple APAs, the correction time was described as the time interval between $\mathrm{T}_{0}$ and the lateral corrector shift, corresponding to the beginning of the effective APA.

- The swing phase started at the end of the APA and finished with heel strike.

Hence, SET was quantified by subtracting S2 from the time of heel strike.

From a methodological point of view, as mentioned above, a MATLAB ${ }^{\circledR}$ script detected events related to the first step and then the evaluator had either to visually confirm the proposed detections or to choose visually more suitable times of events. Heel-off was detected as the instant when the heel velocity in the sagittal plane exceeded the value at baseline (from the start of the trial until S2) by $100 \mathrm{~mm} / \mathrm{s}$ (Ghoussayni et al. 2004; Lambrecht et al. 2017). This algorithm method has been shown to be both 
reliable in comparison to the visual inspection and accurate. Another proposed detection of heel-off was based on the time when the vertical position of the heel marker of the swing leg was beyond the value at baseline (from the start of the trial until S2) plus $1 \mathrm{~mm}$ (Honeine et al. 2016). Furthermore, toe-off was defined as the time of a local maximum in the vertical velocity component of the heel marker, whereas heel strike was associated with the time of a local minimum in the vertical velocity component of the toe marker (Pijnappels et al. 2001). Finally, $\mathrm{T}_{0}$ was defined as the time when mediolateral CoP exceeded mean(baseline) $+1 *$ standard deviation(baseline) just before exceeding mean (baseline) $+3 *$ standard deviation(baseline) (Sun et al. 2015; Sun and Shea 2016). The baseline used was the time interval from the start of the trial to the appearance of the cue in order to avoid postural sway related to the occurrence of visual stimuli as much as possible.

\subsubsection{Electroencephalographic acquisition}

The electroencephalogram was recorded with an $\mathrm{Ag} / \mathrm{AgCl} 128$-scalp-electrode cap (Waveguard ${ }^{\circledR}$, ANT Neuro) positioned according to the 10/05 international system (Oostenveld and Praamstra 2001). Data were acquired with $\mathrm{ASA}^{\mathrm{TM}}$ software (ANT Neuro), using a sampling frequency of $512 \mathrm{~Hz}$ and impedances below $20 \mathrm{k} \Omega$ during the experiment. The data were pre-processed with EEGLAB, a MATLAB ${ }^{\circledR}$ toolbox (Delorme and Makeig 2004). Firstly, EEG data were downsampled at $256 \mathrm{~Hz}$. Then, a low-pass filter was applied in order to remove the first harmonic of the line noise at $100 \mathrm{~Hz}$, as well as a high-pass filter with a cut-off frequency of $0.5 \mathrm{~Hz}$ and a $50-\mathrm{Hz}$ Notch filter for removing, respectively, baseline drift caused, for example, by sweating and line noise. After filtering, only a time interval from $1500 \mathrm{~ms}$ before the first trial to $1000 \mathrm{~ms}$ following the last trial was kept as EEG signal of interest. Next, flat electrodes were automatically removed based on a maximum tolerated flatline duration of $5 \mathrm{~s}$ (using an EEGLAB plugin called clean_rawdata()). The artefact-affected electrodes were, for their part, semi-automatically detected: first, still using clean_rawdata(), a channel was considered abnormal when it was correlated at less than a minimum correlation value to a reconstruction of itself based on other channels in a given time window; second, a final manual rejection of bad electrodes was performed using the EEGLAB plugin trimOutlier(), with lower and upper accepted standard deviation for EEG signal of each channel. Thereafter, a spherical interpolation was performed for identified artefact-affected electrodes, with a maximum interpolation rate of $10 \%(n=13)$ for the whole set of 128 scalp electrodes. The next pre-processing step aimed at removing transient, nonbiological, large-amplitude noise/artifacts (e.g. abrupt impedance changes due to headset motions) using a non-stationary method based on sliding window PCA and implemented in the EEGLAB plugin clean_rawdata(): the Artifact Subspace Reconstruction (ASR) method (Mullen et al. 2015; Chang et al. 2018). The ASR parameter (i.e. the standard deviation cutoff for removal of bursts) was fixed at 20 (as recommended by (Chang et al. 2018)). Average reference was then chosen as re-referencing method. Subsequently, EEG signal during breaks between blocks was removed before using a stationary method 
for decomposing constant fixed-source noise/artefacts/signals of stationary data: the Independent Component Analysis (ICA), and the Infomax algorithm in particular (Delorme et al. 2012). The artefactaffected components that must be removed were automatically selected among the first half of the components thanks to a highly trained IC classifier: ICLabel (Pion-Tonachini et al. 2019). Afterwards, a manual confirmation of the components representing non-neural sources was required and the maximum number of removed IC was fixed at 20. We finally segmented the EEG data either into 2500ms epochs that were time-locked with respect to target onset (1500 ms before and $1000 \mathrm{~ms}$ afterwards; see Online Resource 1 for methodology and results of the target-locked analysis of EEG data) or into 2150-ms epochs that were response-locked (i.e. epochs from $1500 \mathrm{~ms}$ before APA onset until $650 \mathrm{~ms}$ after). A final visual inspection was needed to manually remove epochs containing remaining muscle artefacts or others.

After the manual rejection of epochs of EEG data with artefacts, participants showed trials that were distributed as follows (median (first quartile - third quartile)): 89 (74.25 - 100) congruent trials, 88.5 (75 - 98.25) incongruent trials, and 32 (29 - 35.75), 32 (26 - 38), 83.5 (72.75 - 92), 30 (26.25 - 32.75) trials corresponding respectively to no cue condition, central cue condition, and both valid and invalid spatial cue conditions.

\subsection{ERP data analysis}

Response-locked ERPs (see Online Resource 1 for methodology and results of the target-locked analysis of EEG data) were analysed with the EEGLAB toolbox (Delorme and Makeig 2004), with a baseline interval from 1500 to $1300 \mathrm{~ms}$ before APA onset. The time window analysis was from -800 ms before $\mathrm{S} 2$ to $650 \mathrm{~ms}$ after. In the context of comparison of target conditions as well as cue conditions, we first analysed ERP scalp distribution maps between -400 and $400 \mathrm{~ms}$. Next, based on the observation of topographic maps of ERPs and according to the well-known topography of such ERP components (Shibasaki and Hallett 2006; Berchicci et al. 2012), BP and P300 waves' characteristics were assessed and collected by grand-averaging over the relevant midline electrodes: $\mathrm{FCz}, \mathrm{Cz}, \mathrm{CPz}$ and $\mathrm{Pz}$ for $\mathrm{BP}$ and $\mathrm{Pz}$ for P300. Indeed, we studied movements triggered by external stimuli and, for this reason, late BP and MP components of MRCP were covered by stimulus-evoked activity: P300 was observed instead of late BP and MP, and late P300 seemed to coincide with RAP (Berchicci et al. 2016). BP and P300 peak latencies and amplitudes of all the subjects were compared as a function of the attentional condition. The amplitude of potentials was measured as the difference between the maximum peak of the ERP waveform and the mean baseline voltage. Latency was defined as the interval between APA onset and the point of peak amplitude in the time window of the potential. Time window of BP ranged from -400 to $0 \mathrm{~ms}$ before response onset, whereas time window associated with P300 was between 0 and $400 \mathrm{~ms}$ after APA onset. The grand-averaged ERPs were low-pass filtered at $40 \mathrm{~Hz}$ for displaying purpose only. 
In order to have a more accurate idea of generators of $\mathrm{MRCP}$, we finally performed source localisation (see Online Resource 1 for methodology and detailed results).

\subsection{ERSP data analysis}

ERSP data were also analysed using the EEGLAB toolbox (Delorme and Makeig 2004) with a baseline interval between 1500 and 1100 ms before APA onset (see Online Resource 1 for methodology and results of the target-locked analysis of EEG data). In order to characterise event-related EEG oscillations such as ERD and ERS in theta $(4-7 \mathrm{~Hz})$, alpha $(8-12 \mathrm{~Hz})$ and beta $(13-30 \mathrm{~Hz})$ bands, we applied a timefrequency analysis using a continuous Morlet wavelet transform, with 1.5 cycles at the lowest frequency and 7.875 at highest (factor: 0.3), and analysed ERSP scalp distribution maps between -200 ms before APA onset and $400 \mathrm{~ms}$ after.

\subsection{Statistical analyses}

To evaluate alerting, orienting and conflict resolution effects on behavioural data, a Student's t-test was applied on medians of first step characteristics (SET, RT, APA, swing phase and speed, as well as rates of multiple APAs, incorrect steps and anticipated responses) calculated for each participant in conditions with no cue versus a central cue, with a central cue versus a spatial cue, and with a congruent versus an incongruent target, respectively. When data were not normally distributed, Wilcoxon's signed rank test was carried out. Analyses were performed with SPSS 16.0 software, with the exception of ERP and ERSP analyses (performed with EEGLAB toolbox). Permutation tests for multiple comparisons were performed on overall ERP and ERSP data with FDR correction. Afterwards, conflict resolution, alerting and orienting effects on specific ERP waveforms' features (i.e. peak amplitude and latency) at particular scalp sites were assessed by using the same statistical tests as for behavioural data, with SPSS 16.0 software. The threshold for statistical significance was set to $\mathrm{p}=0.05$.

\section{Results}

\subsection{Behavioural data}

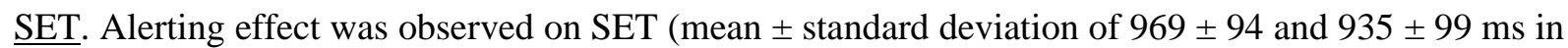
the no cue and central cue conditions, respectively; $\mathrm{p}<0.001)$ and RT $(237 \pm 29$ and $217 \pm 31 \mathrm{~ms}$, respectively; $\mathrm{p}<0.001$ ), but duration of APA (492 \pm 58 and $487 \pm 62 \mathrm{~ms}$, respectively), swing phase (220 \pm 60 and $218 \pm 57 \mathrm{~ms}$, respectively) and speed of first step (934 \pm 128 and $935 \pm 133 \mathrm{~mm} / \mathrm{s}$, respectively) did not significantly differ between cue conditions ( $\mathrm{p}=0.312$ for APA duration, $\mathrm{p}=0.163$ for swing phase and $\mathrm{p}=0.904$ for first step velocity). Besides, orienting effect was not shown on any of the behavioural features of step initiation, as it was the case for validity effect. Furthermore, the SET was significantly 
longer in trials with incongruent flankers than in trials with congruent flankers (979 \pm 91 and $928 \pm 101$ $\mathrm{ms}$, respectively; $\mathrm{p}<0.001)$, as was the APA duration (520 \pm 64 and $470 \pm 58 \mathrm{~ms}$, respectively; $\mathrm{p}<0.001$ ). There was no effect of executive control for the reaction time $(222 \pm 29$ and $224 \pm 29 \mathrm{~ms}$ in the incongruent and congruent conditions, respectively; $\mathrm{p}=0.269)$, the duration of the swing phase (219 \pm 56 and $219 \pm 56 \mathrm{~ms}$, respectively; $\mathrm{p}=0.696)$, or the speed of the first step (936 \pm 129 and $933 \pm 128$ $\mathrm{mm} / \mathrm{s}$, respectively; $\mathrm{p}=0.352$ ).

Multiple APAs. The rate of multiple APAs was significantly higher in trials with incongruent flankers than in trials with congruent flankers (average rates of 39 and $18 \%$, respectively; $p<0.001$ ). Likewise, the duration of multiple APAs was significantly longer in trials with incongruent flankers than in trials with congruent flankers (mean \pm standard deviation of $633 \pm 66$ and $610 \pm 75 \mathrm{~ms}$, respectively; $\mathrm{p}=0.007$ ), as was therefore the SET $(1056 \pm 99$ and $1017 \pm 95 \mathrm{~ms}$, respectively; $\mathrm{p}<0.001)$. Furthermore, this increased SET in the target condition with incongruent flankers may also be explained by a higher RT in this condition (average medians of $199 \pm 23$ and $185 \pm 24 \mathrm{~ms}$, respectively; $\mathrm{p}<0.001$ ). Thanks to a Pearson's partial correlation test, a significant positive association between SET and APA duration in the incongruent condition of target was found, even when controlling for RT $(r=0.787 ; p<0.001)$. However, RT was not significantly correlated to SET after correcting for APA duration $(r=0.372$; $\mathrm{p}=0.172$ ). Finally, there were no alerting, orienting or validity effect on the percentage of multiple APAs in each participant.

Rate of incorrect responses. There was no significant difference in incorrect starts between the flanker conditions ( $0.8 \%$ with congruent flankers, and $0.6 \%$ with incongruent flankers; $\mathrm{p}=0.305)$, but also no alerting, orienting or validity effect $(\mathrm{p}=0.275, \mathrm{p}=0.547$ and $\mathrm{p}=0.313$, respectively).

$\underline{\text { Rate of anticipated responses. Again, no significant difference was found in the rate of anticipated steps }}$ when comparing congruent and incongruent conditions ( 16 and $18 \%$, respectively; $p=0.2$ ). Orienting and validity effects were also absent ( $\mathrm{p}=0.973$ and $\mathrm{p}=0.463$, respectively). Nevertheless, anticipated steps occurred more frequently in trials with a central cue compared to trials without cue (average rates of 18 and $11 \%$, respectively; $\mathrm{p}<0.001)$.

\subsection{Response-locked ERPs}

Neither orientation nor alertness affected response-locked ERPs as seen in Fig. 3. The comparison of topographic maps of response-locked ERPs between both target conditions (Fig. 4) only showed a posterior positive wave included in a time interval ranging from 100 to $400 \mathrm{~ms}$ after APA onset (corresponding in fact to the target-locked P300 (Berchicci et al. 2016)) that was of significantly higher amplitude for the target condition with congruent flankers and lasted longer in the case of incongruent flankers. After analysis of ERPs at Pz, we confirmed that there were no differences in terms of amplitude and latency of BP between the two target conditions. Moreover, the peak latency of the P300 wave was 
not significantly different between both conditions of target, but a significantly higher peak amplitude of P300 was found at Pz for congruent targets (respectively $2.59 \pm 2.28 \mu \mathrm{V}$ vs. $2.31 \pm 2.02 \mu \mathrm{V}$ at Pz; $\mathrm{p}=0.048)$.

It was surprising to have observed a maximal BP at parietal leads while this motor potential is supposed to be symmetrically distributed and maximal at the midline centro-parietal area (Shibasaki and Hallett 2006). Thanks to MRCP source localisation (see Online Resource 1 for further details), we confirmed the location of BP over the sensorimotor cortex, with the supplementary motor area as the main potential generator.

\subsection{Response-locked ERSPs}

Concerning the impact of cueing on response-locked ERSPs (Fig. 5), an alerting effect on posterior alpha and mainly beta ERDs was only seen in a 200-ms time interval before APA onset: trials without a cue were not related to any posterior desynchronisation during this period unlike trials with a neutral or a spatial cue. Based on topographic representations of response-locked EEG spectral activity (Fig. 6a), with a focus on spectral activity at $\mathrm{CPz}$ (Fig. 6b), we found an earlier, of higher amplitude and longer duration beta-band ERD over the sensorimotor cortex for trials with incongruent flankers in comparison with congruent trials. Desynchronisation was also greater and lasted longer in alpha band in incongruent conditions, whereas earlier synchronisation occurred in trials with congruent flankers.

\section{Discussion}

The results of the present study demonstrate that attention can modulate cortical activation during gait initiation in healthy adults. This study is the first to have evaluated the cortical characteristics of attentional modulation (through ANT) during step initiation. Here, we observed that an attentional load does not modify MRCPs but that ERD in alpha and beta bands were modified either by a variation of cue modality (alertness) or target modality (executive control). These cortical oscillations have direct consequences on movement preparation with an effect either on reaction time or on duration of the preparation phase. The absence of an orienting effect in the overall study results will be discussed in the section related to the behavioural results.

\subsection{Effects on cortical activity}

Response-locked ERPs. After having mapped ERPs between $400 \mathrm{~ms}$ before APA onset and $400 \mathrm{~ms}$ after, we observed a centro-parietal negative ERP starting approximately $500 \mathrm{~ms}$ before APA onset and corresponding to the BP. However, neither BP amplitude nor BP latency were significantly different between cue and target conditions. Those results are confirmed by a study of Di Russo and colleagues (Di Russo et al. 2017): they found similar waveforms and topographies for BP associated with "go" and 
"nogo" conditions of a go/nogo paradigm, independently of the presence or not of a warning cue and of a variable or constant inter-stimulus interval. Rather, a reduced BP was observed in the post-error trials (Perri et al. 2016). Another response-locked ERP showed in our study was a centro-parietal positivity occurring between 100 and $400 \mathrm{~ms}$ after APA onset: the response-locked equivalent of target-locked P300 (Berchicci et al. 2016). Response-locked P300 did not significantly differ by its latency between both target conditions (contrary to target-locked P300). It only presented a lower amplitude for trials with incongruent flankers. The greater executive control effect on peak amplitude for responsecompared to target-locked P300 can be explained by the fact that this ERP component seems to be more related to the response than to the stimulus (Berchicci et al. 2016). Indeed, a late component such as P300 reflects a function that bridges perception with response processing and, as found by Berchicci and colleagues, P300 presents a larger amplitude in response- than in stimulus-locked averaging. Finally, the reason why the exogenous components (e.g. P100 and N100) were not visible in the response-locked ERP waveform is because such components are enhanced by stimulus locking (Berchicci et al. 2016). One of the limits of the present study remains the restricted number of trials for some cue conditions related to a few participants, which may weaken the reliability of the results. However, for each subject, the evoked potentials of interest have been clearly observed.

Response-locked ERSPs. Alpha/beta ERD is thought to reflect the activation of regions engaged in visuospatial attention or motor execution (Pfurtscheller, 1999). The alerting effect on the amplitude of alpha and beta ERD in a 200-ms time interval before APA onset is rather related to the phase of sensory processing than associated with step preparation. Moreover, the earlier, of higher amplitude and longer duration alpha- and beta-band ERD observed over the sensorimotor cortex for trials with incongruent flankers instead of congruent ones was in line with the higher task complexity (i.e. higher rate of multiple APAs) and the longer APA duration related to trials with incongruent flankers. ERSPs with target locking were also able to reflect the executive control effect. As reported in studies investigating the standard seated ANT and carried out by Deiber et al. (2013) and Fan et al. (2007), target-locked alphaband ERD was more pronounced as well as of longer duration in trials with incongruent flankers than in trials with congruent flankers - reflecting the greater attentional load. Concerning target-locked betaband oscillations, our results showed that central ERD was more pronounced and lasted longer for incongruent trials than congruent trials in the step initiation ANT (as also reported by Deiber et al. (2013)). This mirrors the specific participation of areas involved in motor preparation and execution.

\subsection{Behavioural effects}

In terms of behavioural characteristics of first step, alertness had an effect on RT, with shorter RT in the presence of a cue just before target appearance in comparison with trials without any cue. This result was associated with a greater rate of anticipated steps in trials with a central cue. It finally led to an alerting effect on SET, with a longer SET related to trials without any cue compared to trials with a 
central cue. SET representing the behavioural reaction time, this observation of an alerting effect on SET confirms a result found previously in studies using the standard seated ANT, that is a significant effect of alertness on RT (Fan et al. 2002, 2005, 2007, 2009; Neuhaus et al. 2010; Deiber et al. 2013; Galvao-Carmona et al. 2014; Williams et al. 2016). Still in line with results from the literature related to the standard ANT, an executive control effect was found on SET, with longer SET in target condition with incongruent flankers compared to congruent trials. This longer SET in conflicting situations can be explained by an increased duration of APA. Nevertheless, unlike our expectations and the orienting effect on RT found in the standard ANT (Fan et al. 2002, 2005, 2007, 2009; Neuhaus et al. 2010; Gamboz et al. 2010; Deiber et al. 2013; Galvao-Carmona et al. 2014; Williams et al. 2016), no orienting effect was exhibited on any of the behavioural features of first step. Besides, a previous study of our group that studied only alerting and orienting effects during step initiation (Tard et al. 2016) has shown significant alerting and orienting effects on RT and SET. One possible reason for the absence of a significant effect of orientation in our present results may be the differences in task design between our version of the ANT and the original version (Fan et al. 2002). With our centrally located spatial cue that gives information concerning the direction of the subsequent target, we assessed rather top-down orienting attention, whereas Fan et al. (2002) and most of the researchers in the field used a spatial cue that was located where the target was subsequently placed on the screen (on the top or bottom), without any indication regarding target direction. The classical ANT thus evaluates bottom-up orienting attention and it may explain why our outcomes differed.

During the step initiation task, we did not record any differences in the rate of incorrect steps as a function of the type of flanker or cue. In contrast, multiple APAs were more frequent and lasted longer in incongruent trials than in congruent trials leading to a longer SET. Presence of multiple APAs reflects the cost of processing conflicting items of information: the CoP shifts contralaterally towards the flankers before being corrected and shifting towards the target, or the stereotyped $\mathrm{CoP}$ displacement is repeated in loop before step execution. It is noteworthy that multiple APAs were also observed in the presence of congruent flankers, as already reported by Uemura et al. (Uemura et al. 2013b). Here, these multiple APAs are probably rather caused by haste than associated with conflict resolution because, in trials with multiple APAs, RT was significantly shorter in congruent trials in comparison with incongruent ones. Furthermore, subjects seem to recover from multiple APAs with more difficulty when a conflict has to be solved. Overall, RT was diminished in trials with multiples APAs compared to trials with usual APAs (mean \pm standard deviation of $192 \pm 22$ and $234 \pm 31 \mathrm{~ms}$, respectively; $\mathrm{p}<0.001$ ). Therefore, haste could represent one of the causes of multiple APAs occurrence, in congruent conditions as well as in conflicting situations. Healthy adults might adopt one of two different strategies: (i) waiting for the identification of the target before choosing the correct motor programme immediately, or (ii) initiating the step by chance or when a large number of arrows appear, and then rectifying the motor programme once it is executed. Thus, the motor programme may be initiated before target identification 
and therefore without having been precisely selected. Adjustments of the motor programme are possible during execution, and might involve rapid, direct sensorimotor loops via visual afferences. Hence, healthy subjects are able to adjust the motor programme after initiation, rather than delaying initiation until the correct motor programme has been selected. It is known that younger adults make fewer APA errors than elderly adults in a choice RT task (Cohen et al. 2011). Our results suggest that multiple APAs do not solely occur under pathological conditions (i.e. when step execution errors are compensated for). In fact, they may well correspond to a physiological phenomenon used in attentional situations, when the motor programme needs to be adapted to environmental constraints. This proposal is supported by a study from Cohen et al. (Cohen et al. 2017) who have found the same amount of multiple APAs in patients with Parkinson's disease compared to healthy controls.

\section{Conclusion}

The simultaneous study of cortical activity and motor behaviour during ANT combined with step initiation allows to better and more fully understand the influence of attentional load on step initiation. In young adults, alertness only impacts the reaction time phase: a longer RT is associated with trials without a cue because a longer time is needed for perceptual discrimination of the subsequent target unlike situations with a cue preceding the target. Indeed, the presence of a cue would stress the sensory perception phase occurring just after target presentation and would lead to an earlier motor preparation. At a cortical level, this alerting effect is reflected into the early target-locked ERP components and ERSPs: a significantly more pronounced target N100 at parietal and occipito-parietal sites and an earlier posterior ERD in alpha band and central-posterior ERD in beta band, in the presence of a cue. Cueing can also result in a higher rate of anticipated responses. The executive control effect leads to longer APAs and thus a longer SET, and to a higher rate a multiple APAs. These behavioural results have an equivalence at the cortical level, with a specific pattern of response-locked ERD in alpha and beta bands over the sensorimotor cortex that clearly illustrates the effect of conflict resolution in young adults. After having led studies in larger populations of healthy adults, this new paradigm combining ANT and step initiation could therefore be used to explore the interaction between attention and step initiation and the neural substrates underlying this interaction in populations with impaired step initiation, such as elderly fallers or patients with Parkinson's disease and freezing of gait. 


\section{Figures}

Fig. 1 Experimental set-up of the modified version of the ANT that is used in the current study

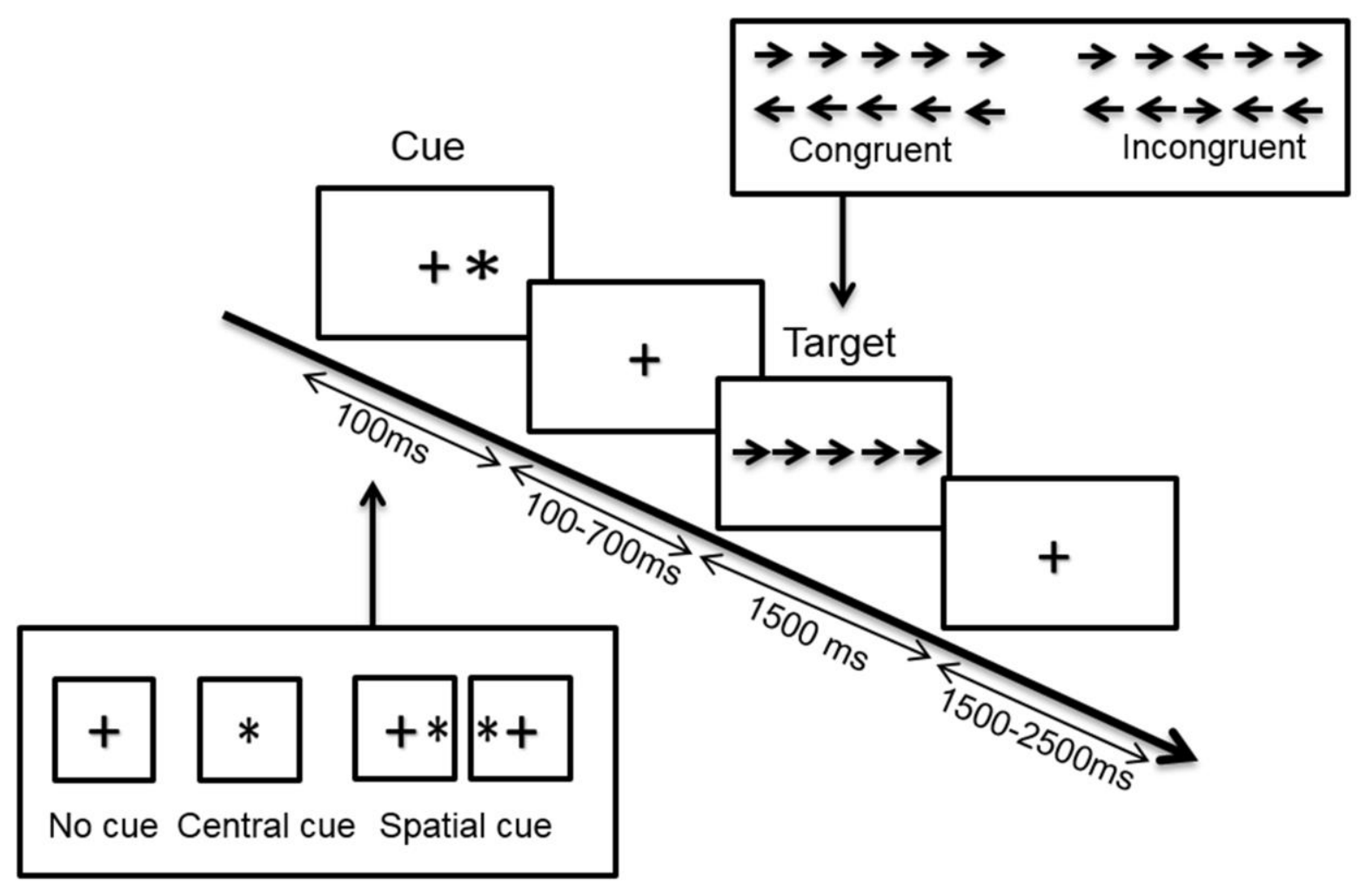


Fig. 2 Step initiation with the right foot, with APA error. Depiction of the step execution time (SET) and its three components: reaction time $R T$ (time interval between the appearance of the target $S 2$ and APA onset $T_{0}$ ), duration of anticipatory postural adjustments $A P A D$ (time interval between $\mathrm{T}_{0}$ and toe-off), and swing phase Swing (from the end of APA to heel strike). The direction of the APA was considered to be normal if the CoP moved backwards and laterally towards the swing foot. An APA error was described as an incorrect CoP displacement. The correction time $C T$ was defined as the time interval between $\mathrm{T}_{0}$ and the lateral corrector shift, corresponding to the beginning of the effective APA. In the figure, the correct APA is preceded by an APA error. CoP (A-P) and CoP (M-L) represent $\mathrm{CoP}$ displacements along the anteroposterior axis and mediolateral axis, respectively. Vertical lines on the horizontal (time) axis indicate (from left to right) S1 (the cue), S2 (the target), heel-off $(H O)$, toe-off $(T O)$, and heel contact $(H C)$

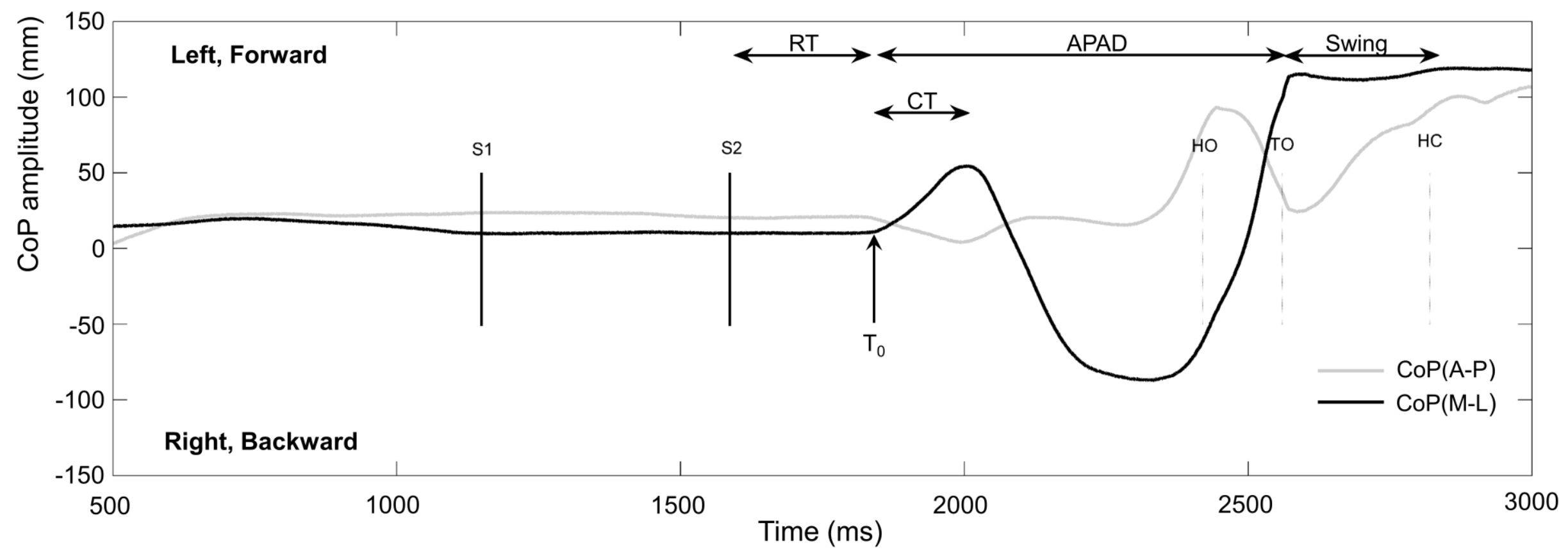


Fig. 3 Influence of cue modality on MRCP. ERP scalp distribution maps between $400 \mathrm{~ms}$ before APA onset and $400 \mathrm{~ms}$ after, for trials with a central cue (first column), an invalid spatial cue (second column), a valid spatial cue (third column) and without cue (fourth column) in the step initiation ANT. Column to the right of ERP scalp distribution maps: significant differences in a permutation test with FDR correction (threshold at $\mathrm{p}<0.05$ ) are marked as red dots 
Central cue Invalid spatial cue Valid spatial cue

No cue $\quad(p<0.05)$ perm with fdr
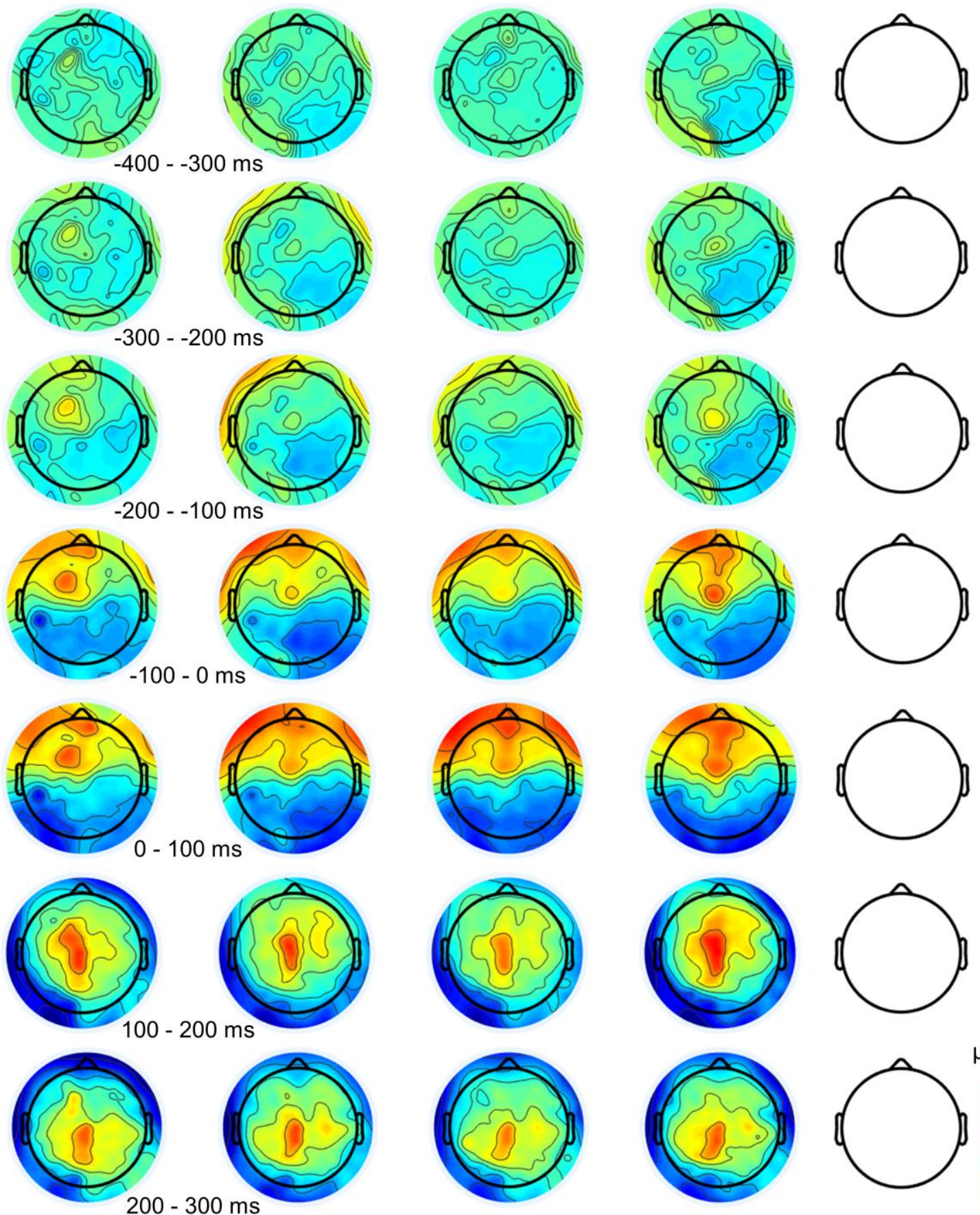

$\mu \mathrm{V}$
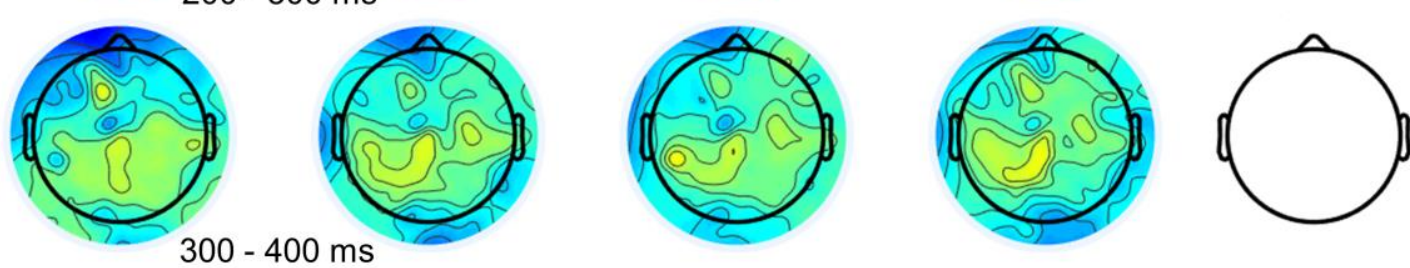

0.3

$-0.6$ 
Fig. 4 Influence of target modality on MRCP. a) ERP scalp distribution maps between $400 \mathrm{~ms}$ before APA onset and $400 \mathrm{~ms}$ after, for trials with congruent flankers (first column) or incongruent flankers (second column) in the step initiation ANT. Column to the right of ERP scalp distribution maps: significant differences in a permutation test with FDR correction (threshold at $\mathrm{p}<0.05$ ) are marked as red dots. b) ERPs at Pz. A negative wave (i.e. the Bereitschaftspotential BP) was observable from around $400 \mathrm{~ms}$ before response onset, followed by a positive wave (corresponding to P300). At the bottom, time intervals highlighted in black represent those for which the p-value related to permutation statistics with FDR correction is significant (i.e. $<0.05$ ). $\mathrm{C}=$ trials with congruent flankers; $\mathrm{I}=$ trials with incongruent flankers

a) Congruent Incongruent $(p<0.05)$ perm with fdr

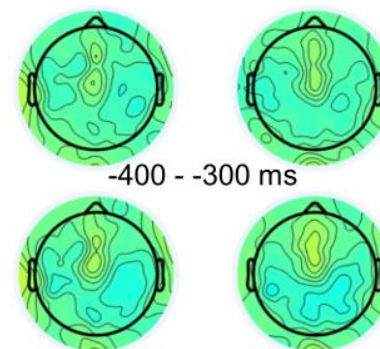

$-300--200 \mathrm{~ms}$
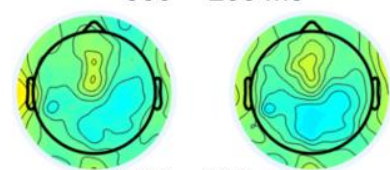

$-200--100 \mathrm{~ms}$

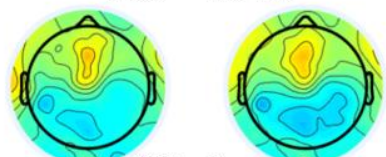

$-100-0 \mathrm{~ms}$
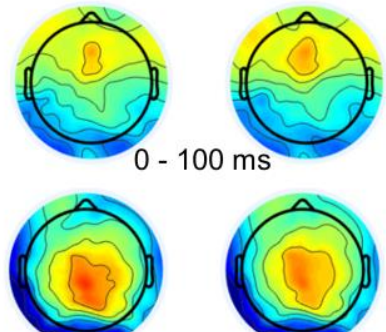

$100-200 \mathrm{~ms}$

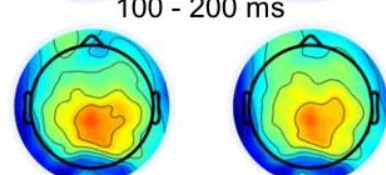

$200-300 \mathrm{~ms}$

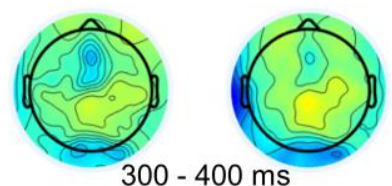

$300-400 \mathrm{~ms}$
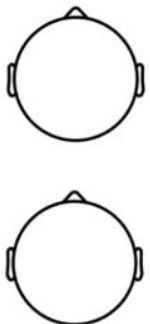

b)
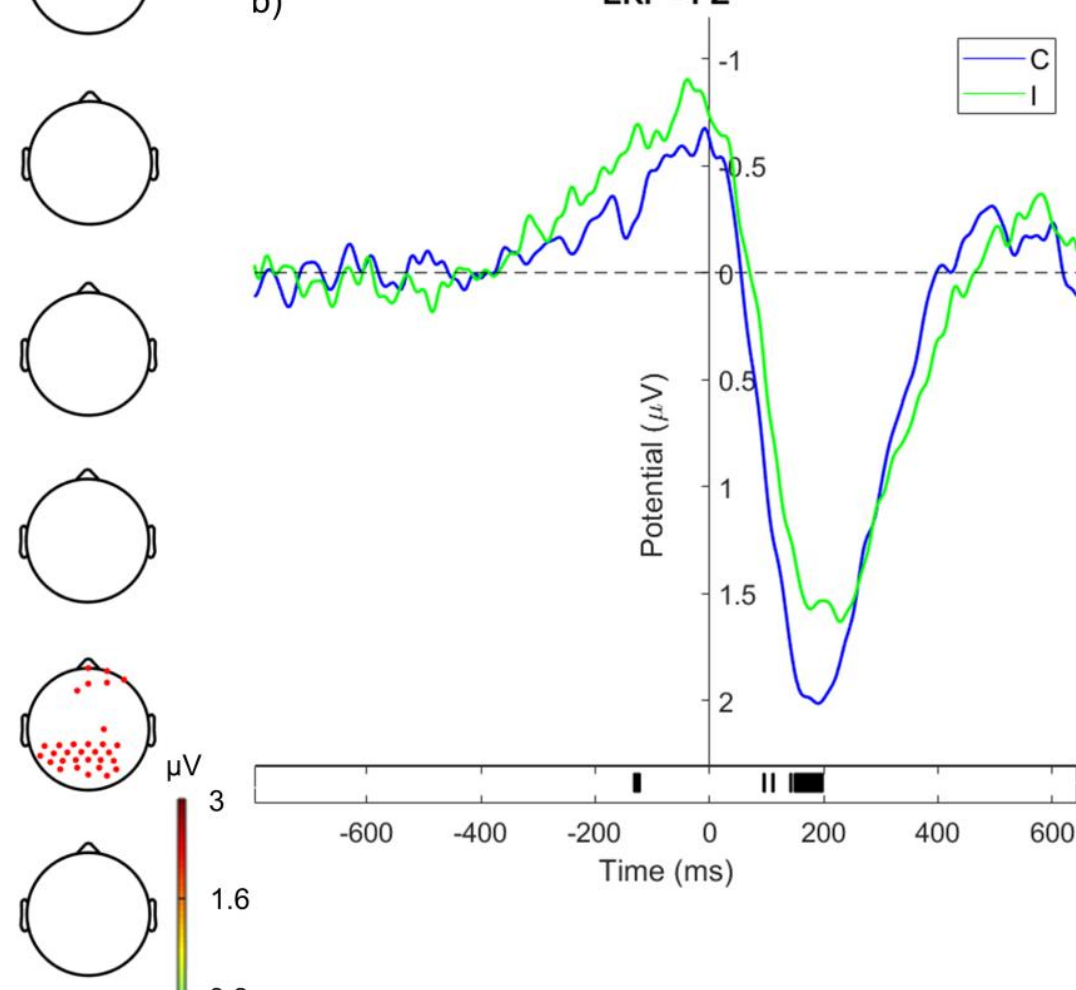

1.6
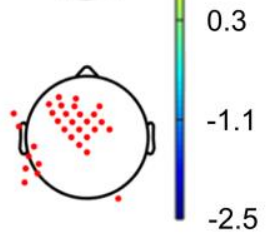
Fig. 5 Influence of cue modality on alpha- and beta-band ERD. Response-locked topographic distributions of time-frequency energy (left: in alpha band (8$12 \mathrm{~Hz}$ ); right: in beta band (13-30 Hz)), corresponding to the four cue conditions (central cue, invalid spatial cue, valid spatial cue and no cue) in the step initiation ANT. Time interval: between -200 ms before APA onset and $400 \mathrm{~ms}$ after. ERD is shown in blue and ERS is shown in red. Column to the right of spectral distributions in each frequency band: significant differences in a permutation test with FDR correction (threshold at $\mathrm{p}<0.05$ ) are marked as red dots 


0000000000
0000000000
0000000000
0000000000
0000000000
0000000000


Fig. 6 Influence of target modality on alpha- and beta-band ERD. a) Response-locked topographic distributions of time-frequency energy (left: in alpha band (8-12 Hz); right: in beta band $(13-30 \mathrm{~Hz})$ ), corresponding to the two flanker conditions (congruent or incongruent) in the step initiation ANT. Time interval: between $200 \mathrm{~ms}$ before response onset and $400 \mathrm{~ms}$ after. b) Response-locked ERSPs at CPz, with onset of APAs aligned with zero on the time axis. ERD is shown in blue and ERS is shown in red. Column to the right of spectral distributions in each frequency band and to the right of spectral distribution among frequency bands from 4 to $30 \mathrm{~Hz}$ at CPz: significant differences in a permutation test with FDR correction (threshold at $\mathrm{p}<0.05$ ) are marked as red dots. $\mathrm{C}=$ trials with congruent flankers; $\mathrm{I}=$ trials with incongruent flankers 
a) ALPHA $(8-12 \mathrm{~Hz})$
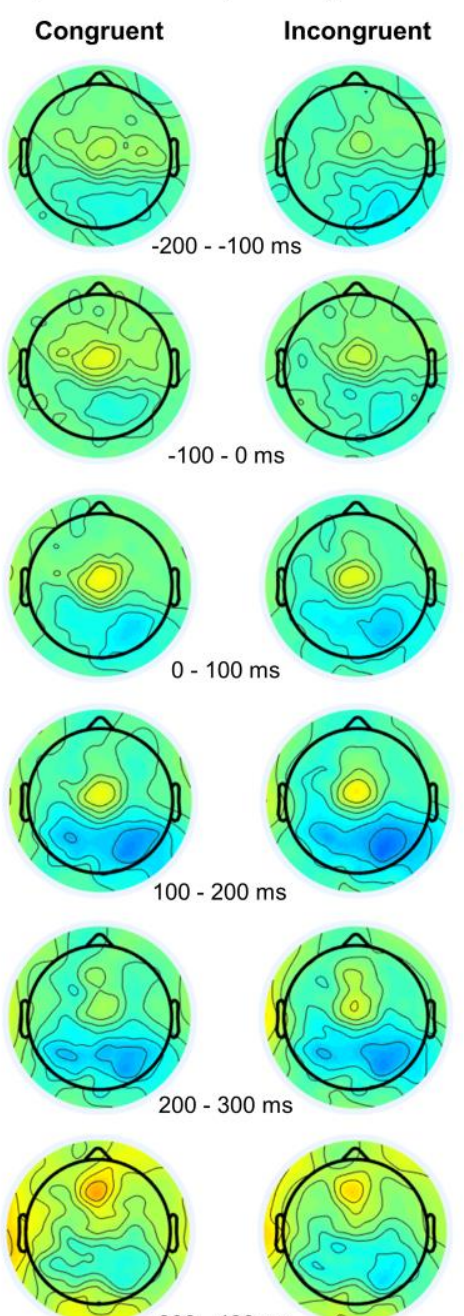

b)
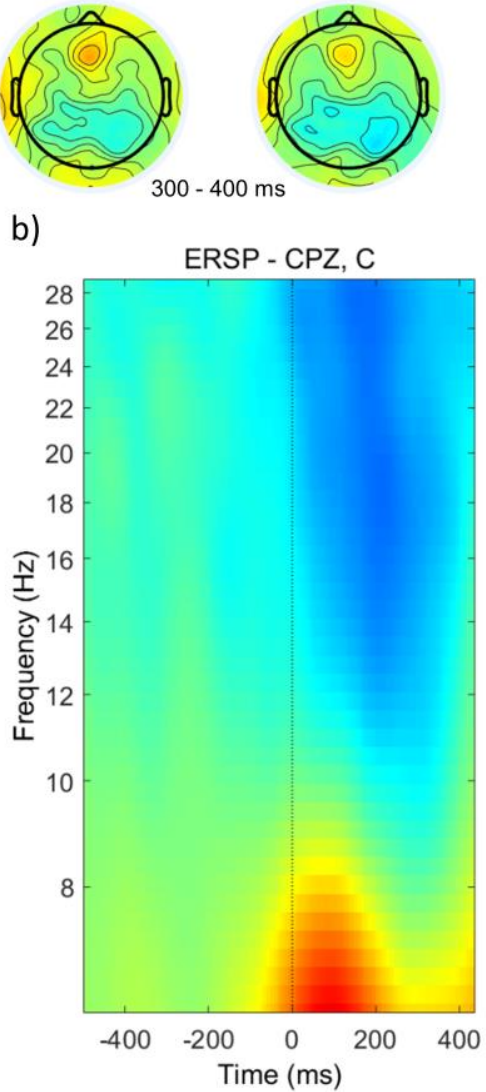

BETA (13-30 Hz)
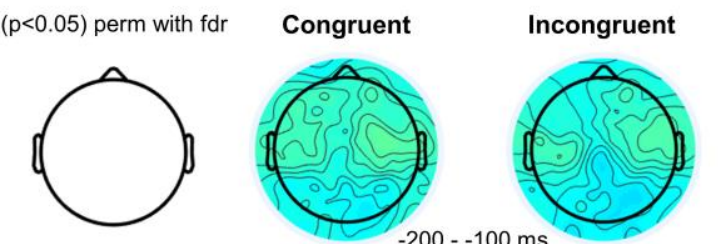

$(p<0.05)$ perm with fdr
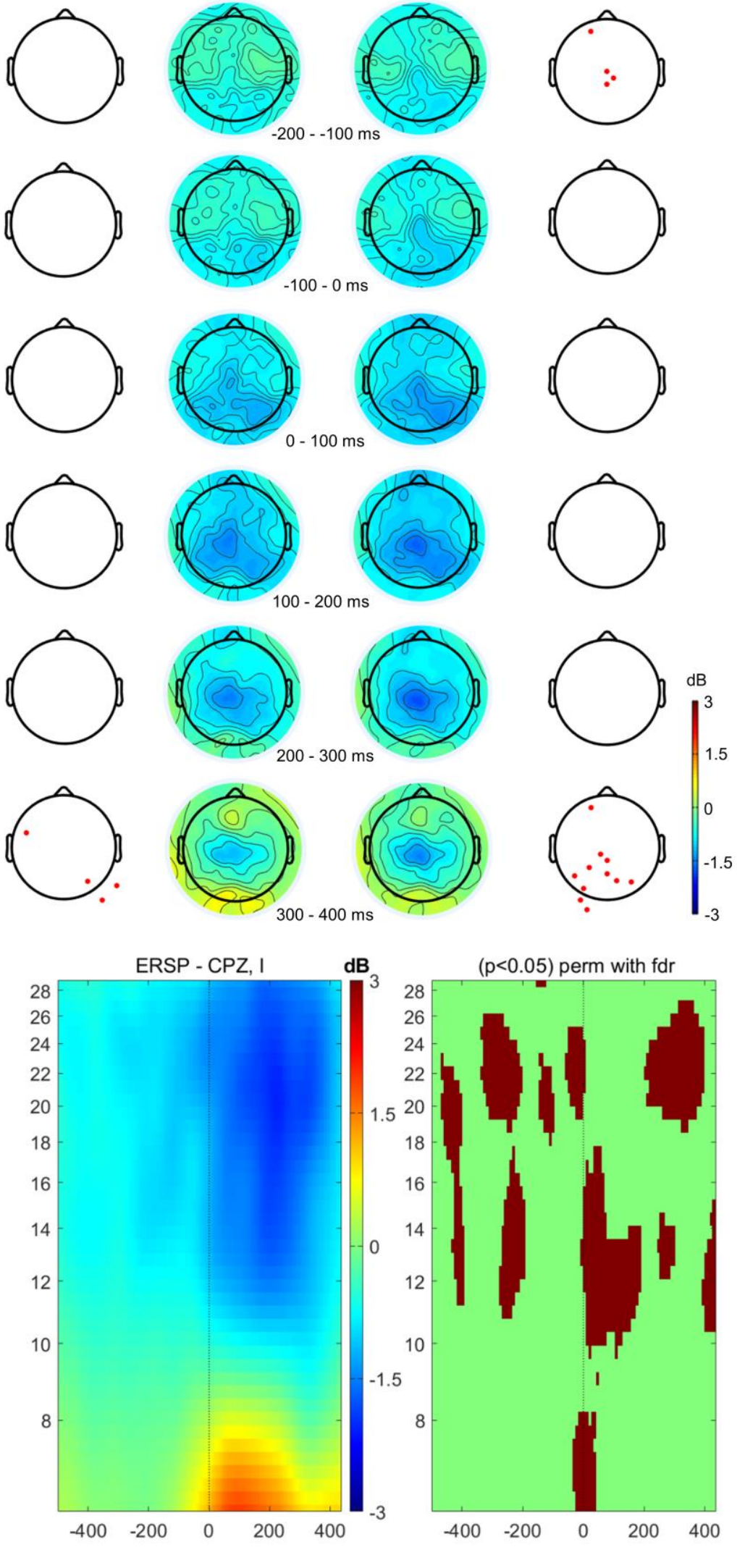
Conflict of interest: Madli Bayot is working on a project that received funding from the European Union's Horizon 2020 research and innovation programme under the Marie Sklodowska-Curie grant agreement No 721577.

Ethical approval: "All procedures performed in studies involving human participants were in accordance with the ethical standards of the institutional and/or national research committee (the Committee for the Protection of Persons North West IV - CPP 15/09, 2015 A00013 46) and with the 1964 Helsinki declaration and its later amendments or comparable ethical standards." 


\section{References}

Berchicci M, Lucci G, Pesce C, et al (2012) Prefrontal hyperactivity in older people during motor planning. NeuroImage 62:1750-1760. https://doi.org/10.1016/j.neuroimage.2012.06.031

Berchicci M, Spinelli D, Di Russo F (2016) New insights into old waves. Matching stimulus- and response-locked ERPs on the same time-window. Biol Psychol 117:202-215. https://doi.org/10.1016/j.biopsycho.2016.04.007

Brenière Y, Do MC (1991) Control of gait initiation. J Mot Behav 23:235-240. https://doi.org/10.1080/00222895.1991.9942034

Chang C-Y, Hsu S-H, Pion-Tonachini L, Jung T-P (2018) Evaluation of Artifact Subspace Reconstruction for Automatic EEG Artifact Removal. Conf Proc Annu Int Conf IEEE Eng Med Biol Soc IEEE Eng Med Biol Soc Annu Conf 2018:1242-1245. https://doi.org/10.1109/EMBC.2018.8512547

Cohen MX, Ridderinkhof KR (2013) EEG source reconstruction reveals frontal-parietal dynamics of spatial conflict processing. PloS One 8:e57293. https://doi.org/10.1371/journal.pone.0057293

Cohen RG, Nutt JG, Horak FB (2011) Errors in postural preparation lead to increased choice reaction times for step initiation in older adults. J Gerontol A Biol Sci Med Sci 66:705-713. https://doi.org/10.1093/gerona/glr054

Cohen RG, Nutt JG, Horak FB (2017) Recovery from Multiple APAs Delays Gait Initiation in Parkinson's Disease. Front Hum Neurosci 11:. https://doi.org/10.3389/fnhum.2017.00060

de Souza Fortaleza AC, Mancini M, Carlson-Kuhta P, et al (2017) Dual task interference on postural sway, postural transitions and gait in people with Parkinson's disease and freezing of gait. Gait Posture 56:76-81. https://doi.org/10.1016/j.gaitpost.2017.05.006

Deiber M-P, Ibañez V, Missonnier P, et al (2013) Age-associated modulations of cerebral oscillatory patterns related to attention control. NeuroImage 82:531-546. https://doi.org/10.1016/j.neuroimage.2013.06.037

Delorme A, Makeig S (2004) EEGLAB: an open source toolbox for analysis of single-trial EEG dynamics including independent component analysis. J Neurosci Methods 134:9-21. https://doi.org/10.1016/j.jneumeth.2003.10.009

Delorme A, Palmer J, Onton J, et al (2012) Independent EEG Sources Are Dipolar. PLOS ONE 7:e30135. https://doi.org/10.1371/journal.pone.0030135

Delval A, Braquet A, Dirhoussi N, et al (2018) Motor Preparation of Step Initiation: Error-related Cortical Oscillations. Neuroscience 393:12-23. https://doi.org/10.1016/j.neuroscience.2018.09.046

Delval A, Dujardin K, Tard C, et al (2012) Anticipatory Postural Adjustments During Step Initiation: Elicitation by Auditory Stimulation of Differing Intensities. Neuroscience 219:166-174. https://doi.org/10.1016/j.neuroscience.2012.05.032

Di Russo F, Berchicci M, Bozzacchi C, et al (2017) Beyond the "Bereitschaftspotential": Action preparation behind cognitive functions. Neurosci Biobehav Rev 78:57-81. https://doi.org/10.1016/j.neubiorev.2017.04.019 
Fan J, Byrne J, Worden MS, et al (2007) The relation of brain oscillations to attentional networks. J Neurosci Off J Soc Neurosci 27:6197-6206. https://doi.org/10.1523/JNEUROSCI.183307.2007

Fan J, Gu X, Guise KG, et al (2009) Testing the behavioral interaction and integration of attentional networks. Brain Cogn 70:209-220. https://doi.org/10.1016/j.bandc.2009.02.002

Fan J, McCandliss BD, Fossella J, et al (2005) The activation of attentional networks. NeuroImage 26:471-479. https://doi.org/10.1016/j.neuroimage.2005.02.004

Fan J, McCandliss BD, Sommer T, et al (2002) Testing the efficiency and independence of attentional networks. J Cogn Neurosci 14:340-347. https://doi.org/10.1162/089892902317361886

Galvao-Carmona A, González-Rosa JJ, Hidalgo-Muñoz AR, et al (2014) Disentangling the attention network test: behavioral, event related potentials, and neural source analyses. Front Hum Neurosci 8:813. https://doi.org/10.3389/fnhum.2014.00813

Gamboz N, Zamarian S, Cavallero C (2010) Age-related differences in the attention network test (ANT). Exp Aging Res 36:287-305. https://doi.org/10.1080/0361073X.2010.484729

Ghoussayni S, Stevens C, Durham S, Ewins D (2004) Assessment and validation of a simple automated method for the detection of gait events and intervals. Gait Posture 20:266-272. https://doi.org/10.1016/j.gaitpost.2003.10.001

Honeine J-L, Schieppati M, Crisafulli O, Do M-C (2016) The Neuro-Mechanical Processes That Underlie Goal-Directed Medio-Lateral APA during Gait Initiation. Front Hum Neurosci 10:. https://doi.org/10.3389/fnhum.2016.00445

Jankelowitz SK, Colebatch JG (2002) Movement-related potentials associated with self-paced, cued and imagined arm movements. Exp Brain Res 147:98-107. https://doi.org/10.1007/s00221-002$1220-8$

Jian Y, Winter D, Ishac M, Gilchrist L (1993) Trajectory of the body COG and COP during initiation and termination of gait. Gait Posture 1:9-22. https://doi.org/10.1016/0966-6362(93)90038-3

Lajoie Y, Teasdale N, Bard C, Fleury M (1996) Attentional demands for walking: Age-related changes. In: Changes in sensory motor behavior in aging. Elsevier Science, New York, NY, US, pp 235256

Lambrecht S, Harutyunyan A, Tanghe K, et al (2017) Real-Time Gait Event Detection Based on Kinematic Data Coupled to a Biomechanical Model. Sensors 17:. https://doi.org/10.3390/s17040671

Lord SR, Fitzpatrick RC (2001) Choice Stepping Reaction TimeA Composite Measure of Falls Risk in Older People. J Gerontol Ser A 56:M627-M632. https://doi.org/10.1093/gerona/56.10.M627

Melinščak F, Montesano L, Minguez J (2014) Discriminating Between Attention and Mind Wandering During Movement Using EEG

Melinscak F, Montesano L, Minguez J (2016) Asynchronous detection of kinesthetic attention during mobilization of lower limbs using EEG measurements. J Neural Eng 13:016018. https://doi.org/10.1088/1741-2560/13/1/016018

Mullen TR, Kothe CAE, Chi YM, et al (2015) Real-Time Neuroimaging and Cognitive Monitoring Using Wearable Dry EEG. IEEE Trans Biomed Eng 62:2553-2567. https://doi.org/10.1109/TBME.2015.2481482 
Neuhaus AH, Koehler S, Opgen-Rhein C, et al (2007) Selective anterior cingulate cortex deficit during conflict solution in schizophrenia: an event-related potential study. J Psychiatr Res 41:635-644. https://doi.org/10.1016/j.jpsychires.2006.06.012

Neuhaus AH, Urbanek C, Opgen-Rhein C, et al (2010) Event-related potentials associated with Attention Network Test. Int J Psychophysiol Off J Int Organ Psychophysiol 76:72-79. https://doi.org/10.1016/j.ijpsycho.2010.02.005

Oostenveld R, Praamstra P (2001) The five percent electrode system for high-resolution EEG and ERP measurements. Clin Neurophysiol Off J Int Fed Clin Neurophysiol 112:713-719

Perri RL, Berchicci M, Lucci G, et al (2016) How the brain prevents a second error in a perceptual decision-making task. Sci Rep 6:32058. https://doi.org/10.1038/srep32058

Petersen SE, Posner MI (2012) The attention system of the human brain: 20 years after. Annu Rev Neurosci 35:73-89. https://doi.org/10.1146/annurev-neuro-062111-150525

Pfurtscheller G (1999) Event-related EEG/MEG synchronization and desynchronization: basic principles. Clin Neurophysiol Off J Int Fed Clin Neurophysiol 110:1842-1857

Pfurtscheller G, Neuper C, Andrew C, Edlinger G (1997) Foot and hand area mu rhythms. Int J Psychophysiol Off J Int Organ Psychophysiol 26:121-135

Pfurtscheller G, Zalaudek K, Neuper C (1998) Event-related beta synchronization after wrist, finger and thumb movement. Electroencephalogr Clin Neurophysiol 109:154-160

Pijnappels M, Bobbert MF, van Dieën JH (2001) Changes in walking pattern caused by the possibility of a tripping reaction. Gait Posture 14:11-18

Pion-Tonachini L, Kreutz-Delgado K, Makeig S (2019) ICLabel: An automated electroencephalographic independent component classifier, dataset, and website. NeuroImage 198:181-197. https://doi.org/10.1016/j.neuroimage.2019.05.026

Posner MI, Petersen SE (1990) The attention system of the human brain. Annu Rev Neurosci 13:25-42. https://doi.org/10.1146/annurev.ne.13.030190.000325

Raz A, Buhle J (2006) Typologies of attentional networks. Nat Rev Neurosci 7:367-379. https://doi.org/10.1038/nrn1903

Shibasaki H (2012) Cortical activities associated with voluntary movements and involuntary movements. Clin Neurophysiol Off J Int Fed Clin Neurophysiol 123:229-243. https://doi.org/10.1016/j.clinph.2011.07.042

Shibasaki H, Hallett M (2006) What is the Bereitschaftspotential? Clin Neurophysiol Off J Int Fed Clin Neurophysiol 117:2341-2356. https://doi.org/10.1016/j.clinph.2006.04.025

Sparto PJ, Fuhrman SI, Redfern MS, et al (2014) Postural adjustment errors during lateral step initiation in older and younger adults. Exp Brain Res 232:3977-3989. https://doi.org/10.1007/s00221014-4081-z

Sun R, Guerra R, Shea JB (2015) The posterior shift anticipatory postural adjustment in choice reaction step initiation. Gait Posture 41:894-898. https://doi.org/10.1016/j.gaitpost.2015.03.010

Sun R, Shea JB (2016) Probing attention prioritization during dual-task step initiation: a novel method. Exp Brain Res 234:1047-1056. https://doi.org/10.1007/s00221-015-4534-z 
Suzuki M, Miyai I, Ono T, et al (2004) Prefrontal and premotor cortices are involved in adapting walking and running speed on the treadmill: an optical imaging study. NeuroImage 23:1020-1026. https://doi.org/10.1016/j.neuroimage.2004.07.002

Suzuki M, Miyai I, Ono T, Kubota K (2008) Activities in the frontal cortex and gait performance are modulated by preparation. An fNIRS study. NeuroImage 39:600-607. https://doi.org/10.1016/j.neuroimage.2007.08.044

Tard C, Dujardin K, Bourriez J-L, et al (2013) Stimulus-driven attention modulates the release of anticipatory postural adjustments during step initiation. Neuroscience 247C:25-34. https://doi.org/10.1016/j.neuroscience.2013.05.015

Tard C, Dujardin K, Girard A, et al (2016) How does visuospatial attention modulate motor preparation during gait initiation? Exp Brain Res 234:39-50. https://doi.org/10.1007/s00221-015-4436-0

Uemura K, Oya T, Uchiyama Y (2013a) Effects of visual interference on initial motor program errors and execution times in the choice step reaction. Gait Posture 38:68-72. https://doi.org/10.1016/j.gaitpost.2012.10.016

Uemura K, Oya T, Uchiyama Y (2013b) Effects of speed and accuracy strategy on choice step execution in response to the flanker interference task. Hum Mov Sci 32:1393-1403. https://doi.org/10.1016/j.humov.2013.07.007

Uemura K, Yamada M, Nagai K, et al (2012) Effects of dual-task switch exercise on gait and gait initiation performance in older adults: preliminary results of a randomized controlled trial. Arch Gerontol Geriatr 54:e167-171. https://doi.org/10.1016/j.archger.2012.01.002

van den Bogert AJ, Pavol MJ, Grabiner MD (2002) Response time is more important than walking speed for the ability of older adults to avoid a fall after a trip. J Biomech 35:199-205

Vidailhet M, Stocchi F, Rothwell JC, et al (1993) The Bereitschaftspotential preceding simple foot movement and initiation of gait in Parkinson's disease. Neurology 43:1784-1788

Williams RS, Biel AL, Wegier P, et al (2016) Age differences in the Attention Network Test: Evidence from behavior and event-related potentials. Brain Cogn 102:65-79. https://doi.org/10.1016/j.bandc.2015.12.007 